\title{
ERRATUM
}

\section{On the homeotopy group of a non-orientable surface}

\author{
By JOAN S. BIRMAN AND D. R. J. CHILLINGWORTH
}

(Received 4 July 2003)

The purpose of this note is to call attention to an error in the paper [1]. The main result was a new proof of the main theorem in [2]. In line 1 of page 446 of [1] one finds the following remark: "This is the result obtained in [2]". However, a comparison of the two results shows that the generator which is called $d_{1} d_{2}^{-1}$ in the notation of [1] appears in [2] but is missing from the list in [1].

The source of the omission in [1] is in the last line on page 439 of [1], where the relations which are written $j d_{i} j^{-1}=d_{i}^{-1}, i=1,2$ should have been written $j d_{i} j^{-1}=d_{k}^{-1}$, $\{i, k\}=\{1,2\}$. This resulted in the omission of the generator $d_{1} d_{2}^{-1}$ from Lemma 1 and Theorem 2 . The error affected only the case $g=4$ because for $g$ even and greater than 4 the well-known 'lantern relation' can be used to omit the extra generator.

The authors are grateful to M. Korkmaz, who pointed out the gap to us in 1998. Unfortunately, we neglected to submit an erratum at that time. We knew that Kormaz had cited [2] in his work. We hoped that future authors, if any, would spot the error in a similar way, as it had not caused a problem in 29 years. Unfortunately, however, 5 years later B. Szepietowski used the methods of [1] and failed to check its calculations against those in [2] when he wrote his paper [4]. While the oversight did not lead to an error in his result, it did lead to questions about the accuracy of his proof, which we regret. We now realize that this erratum is needed to set the record straight.

\section{REFERENCES}

[1] Joan S. Birman and D. R. J. Chillingworth. On the homeotopy group of a non-orientable surface. Proc. Camb. Phil. Soc. 71 (1972), 437-448.

[2] D. R. J. Chillingworth. A finite set of generators for the homeotopy group of a non-orientable surface. Proc. Camb. Phil. Soc. 65 (1969), 409-430.

[3] M. Korkмaz. First homology group of mapping class groups of non-orientable surfaces. Math. Proc. Camb. Phil. Soc. 123 (1998), 487-499.

[4] B. Szepietowski. Mapping class group of a non-orientable surface and moduli space of Klein surfaces. C.R. Acad. Sci. Paris, Ser I, 335 (2002), 1053-1056.

Joan S. Birman, Department of Mathematics, Barnard College of Columbia University, New York, New York 10027, e-mail: jb@math.columbia.edu.

D. R. J. Chillingworth, School of Mathematics, University of Southampton, Southampton SO17 1BJ, UK, e-mail: D.R.J.Chillingworth@maths.soton.ac.uk. 